\title{
Molecule Formation in Optical Lattice Wells by Resonantly Modulated Magnetic Fields
}

\author{
Jesper Fevre Bertelsen and Klaus Mølmer \\ Danish National Research Foundation Center for Quantum Optics \\ Department of Physics and Astronomy, University of Aarhus \\ DK 8000 Aarhus C, Denmark
}

(Dated: 20th November 2018)

\begin{abstract}
We present a theoretical model for formation of molecules in an optical lattice well where a resonant coupling of atomic and molecular states is provided by small oscillations of a magnetic field in the vicinity of a Feshbach resonance. As opposed to an adiabatic sweep over the full resonance, this provides a coherent coupling with a frequency that can be tuned to meet resonance conditions in the system. The effective Rabi frequencies for this coupling are calculated and simulations show perfect Rabi oscillations. Robust production of molecules with an adiabatic sweep of the modulation frequency is demonstrated. For very large oscillation amplitudes, the Rabi oscillations are distorted but still effective and fast association is possible.
\end{abstract}

PACS numbers: 03.75.Nt, 03.75.Ss, 36.90.+f

Creation of molecules from ultracold atoms has been successfully achieved by making an adiabatic sweep of the magnetic field across a Feshbach resonance [1, 2, 3, 4, 5. 6]. Feshbach resonances can be used to create molecules, because of the transformation of a scattering state into a new bound state, when the scattering length changes sign. This occurs when the magnetic field varies near a Feshbach resonance [7]:

$$
a_{\mathrm{sc}}(B)=a_{\mathrm{bg}}\left(1-\frac{\Delta}{B-B_{0}}\right) .
$$

Here $a_{\mathrm{bg}}$ is the background value of the scattering length, and $B_{0}$ and $\Delta$ are the location and width of the resonance. In a recent publication [8] it was demonstrated that near Feshbach resonances, molecules can be created by harmonic modulation of the magnetic field:

$$
B(t)=B^{\prime}+b \sin \left(\omega_{B} t\right),
$$

where $\omega_{B}$ is resonant with the energy difference between the atomic and the molecular state at the magnetic field $B^{\prime}$. It is the purpose of this article to analyze this process for two atoms in an optical lattice well, which is a very clean system for which many-body effects can be neglected. One argument in favour of producing the molecules in the lattice resonantly rather than by sweeping across the Feshbach resonance is that it enables the preparation of well-controlled coherent superposition states of atomic and molecular components.

Ultracold molecules in optical lattices have been created by photoassociation [9, 10], and proposals for creating heteronuclear molecules have also been presented 11. In 10], Rabi oscillations have been seen with this method. In 12, 13 the production of molecules via Feshbach resonances in a $1 \mathrm{D}$ confining lattice potential has been studied and very recently the first successful creation of molecules in separate wells of a 3-dimensional optical lattice by sweeping across a Feshbach resonance has been reported 14]. As it turns out these molecules can be very long-lived (up to $700 \mathrm{~ms}$ ) since they do not suffer from decay due to inelastic collisions.

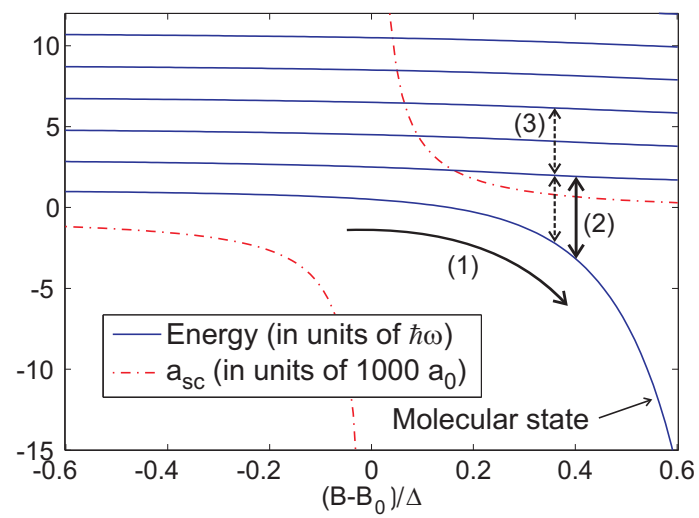

Figure 1: The $s$-wave spectrum of the potential (3) around the $B_{0}=155 \mathrm{G}$ Feshbach resonance in ${ }^{85} \mathrm{Rb}$ in a harmonic oscillator trap with $\omega=2 \pi \cdot 30 \mathrm{kHz}$. The variation of the scattering length $a_{\text {sc }}$ as a function of $\mathrm{B}$ is shown as a dot-dashed line. Note that the bound (molecular) state with negative energy occurs in the $a>0$ (repulsive effective interaction) regime. The typical adiabatic production of molecules by a magnetic field scan across the resonance is indicated by the arrow (1). Arrow (2) illustrates the production of molecules by resonant association as analyzed in the present article, while the dotted arrows (3) show an accidental three-level resonance (discussed in the text).

In [8] a commonly used resonance in ${ }^{85} \mathrm{Rb}$ is applied. It has the parameters [15] $B_{0}=155.041 \mathrm{G}, \Delta=10.71 \mathrm{G}$, and $a_{\mathrm{bg}}=-443 a_{0}$, where $a_{0}$ is the Bohr radius. This resonance has previously been used to produce molecules [1] and to tune the scattering length to make stable atomic BECs of ${ }^{85} \mathrm{Rb}$ atoms [16].

We now look at a simple two-atom model for molecule formation in a three-dimensional optical lattice which is sufficiently deep that the atoms do not tunnel between 
lattice sites. The system may either be in the Mott insulating state [17], or we may have a mean field state with number fluctuations in the site occupancy, in which case the collisional interactions apply to the two-atom component of the state on each site [18]. The assumption is that we have two identical atoms in a lattice well and neglect couplings to atoms in other wells. The lattice potential is $V(x, y, z)=V_{0} \sum_{i=x, y, z} \sin ^{2}\left(k x_{i}\right), \quad k=\frac{2 \pi}{\lambda}$, where $\lambda$ is the wavelength of the standing wave laser field of the lattice, and we make the harmonic and isotropic approximation in each well: $V(x, y, z) \simeq \frac{1}{2} m \omega^{2} \sum_{i=x, y, z} x_{i}^{2}$. The potential for two atoms in this harmonic potential decouples into parts describing the center-of-mass and the relative motion. The interaction between atoms is modelled by the Huang s-wave pseudopotential [19] and the total potential for the relative motion becomes

$$
V(\mathbf{r})=\frac{1}{2}(m / 2) \omega^{2} r^{2}+\frac{4 \pi \hbar^{2} a_{\mathrm{sc}}(B)}{m} \delta^{(3)}(\mathbf{r}) \frac{\partial}{\partial r} r
$$

This is an adequate approximation to the more comprehensive analysis [20] which takes into account the spatial confinement and the energy dependence of the scattering length. We note that a more precise treatment amounts in principle to the application of the exact energy eigenvalues and matrix elements instead of the analytical expressions offered by the pseudo-potential in (3). Such an analysis will lead to the same physical behaviour of the system. For our examples with Rb atoms and typical optical lattice parameters, the characteristic values of the scattering length is about one order of magnitude smaller than the harmonic oscillator length, and we expect that the analytical model provides a reasonable approximation to the exact dynamics 21].

We adopt harmonic oscillator units $\sqrt{\hbar /(m / 2) \omega}$ for length and $\hbar \omega$ for energy, and we express the energy of the s-wave states by a generalized harmonic oscillator quantum number: $E_{i}(B)=2 \nu_{i}(B)+3 / 2$. Following $[22$, 23], $\nu_{i}(B)$ are obtained as the solutions of the equation

$$
\frac{2 \Gamma\left(-\nu_{i}\right)}{\Gamma\left(-\nu_{i}-1 / 2\right)}=\frac{1}{a_{\mathrm{sc}}(B)}
$$

where $\Gamma$ is the gamma function. The energy spectrum is plotted in Fig. 11 for the ${ }^{85} \mathrm{Rb}$ resonance.

Assuming a time dependent magnetic field, the eigenstates depend on $a_{\mathrm{sc}}$ and thus on $t$. We shall label the states $\mid \psi_{n}(t)>, n=0,1,2, \ldots$ with increasing energy. They will at every instant form a complete set. Expanding the wave function $\mid \psi(t)>$ on these states we obtain the following equations for the expansion coefficients $c_{m}$ :

$$
i \hbar \frac{d c_{m}(t)}{d t}=E_{m}(t) c_{m}(t)-\left.i \hbar \sum_{n=0}^{\infty} \frac{\partial O_{m n}\left(t, t^{\prime}\right)}{\partial t^{\prime}}\right|_{t^{\prime}=t} c_{n}(t)
$$

where $O_{m n}\left(t, t^{\prime}\right) \equiv<\psi_{m}(t) \mid \psi_{n}\left(t^{\prime}\right)>$. Introducing the $\nu$-values $\nu_{m}$ and $\nu_{n}$ corresponding to $\psi_{m}(t)$ and $\psi_{n}\left(t^{\prime}\right)$ respectively and by following a trick in [22], or by explicitly evaluating the integral of the wave functions [24], we obtain

$$
\begin{aligned}
O_{m n}\left(t, t^{\prime}\right)=\frac{f\left(\nu_{m}\right) f\left(\nu_{n}\right)}{\nu_{n}-\nu_{m}} \\
\cdot \frac{\Gamma\left(-\nu_{m}\right) \Gamma\left(-\nu_{n}-\frac{1}{2}\right)-\Gamma\left(-\nu_{n}\right) \Gamma\left(-\nu_{m}-\frac{1}{2}\right)}{\sqrt{\Gamma\left(-\nu_{m}-\frac{1}{2}\right) \Gamma\left(-\nu_{m}\right)} \cdot \sqrt{\Gamma\left(-\nu_{n}-\frac{1}{2}\right) \Gamma\left(-\nu_{n}\right)}} \\
=\frac{f\left(\nu_{m}\right) f\left(\nu_{n}\right)}{\nu_{n}-\nu_{m}}\left(\sqrt{\frac{a_{\mathrm{sc}}\left(t^{\prime}\right)}{a_{\mathrm{sc}}(t)}}-\sqrt{\frac{a_{\mathrm{sc}}(t)}{a_{\mathrm{sc}}\left(t^{\prime}\right)}}\right)
\end{aligned}
$$

where

$$
f(\nu)=\frac{1}{\sqrt{\Psi(-\nu)-\Psi\left(-\nu-\frac{1}{2}\right)}}
$$

and $\Psi(\nu) \equiv \frac{d}{d \nu} \ln (\Gamma(\nu))$ is the digamma function.

Inserting now the oscillating magnetic field (2) in (5) we get the following coupling between the states:

$$
i \hbar \frac{d c_{m}(t)}{d t}=E_{m}(t) c_{m}(t)+i \hbar \cos \left(\omega_{B} t\right) \sum_{n=0}^{\infty} \Omega_{m n}(t) c_{n}(t)
$$

where the Rabi frequencies $\Omega_{m n}$ are given by

$$
\Omega_{m n}=\left.\frac{-(b / \Delta) \omega_{B} \Delta^{2}}{\left(B-B_{0}\right)\left(B-B_{0}-\Delta\right)} f\left(\nu_{n}\right)^{2} \frac{\partial O_{\nu_{m}, \nu_{n}^{\prime}}}{\partial \nu_{n}^{\prime}}\right|_{\nu_{n}^{\prime}=\nu_{n}}
$$

The Rabi frequencies are proportional to $b / \Delta$, and the rest of the expression is plotted in Fig. 2 We remark that $\Omega_{n n}=0$. Equivalent to the radiative coupling of atomic energy levels, if $\hbar \omega_{B}$ matches the energy difference between two specific levels $m$ and $n$, Rabi oscillations between these two levels with a constant frequency $\Omega_{m n}$ are expected as long as $b \ll \Delta$. Notice that due to the factor $\omega_{B}$ in Eq. 9] there is a much stronger resonant coupling to the lowest (molecular) state than among the atomic states on the molecular side of the resonance.

Using Eq. (5) we have calculated the dynamics of the resonant association of two ${ }^{85} \mathrm{Rb}$ atoms in an $\omega=2 \pi \cdot 30$ $\mathrm{kHz}$ lattice well. Referring to the above resonance we use $B^{\prime}=B_{0}+0.4 \Delta=159.33 \mathrm{G}$, and $b=0.01$ and we start out in the lowest atomic state (on the $2^{\text {nd }}$ lowest curve in Fig. 1). The expected resonant frequency is found from (4): $\omega_{B}^{\text {res }}=\left(E_{1}\left(B^{\prime}\right)-E_{0}\left(B^{\prime}\right)\right) / \hbar=5.079 \omega=2 \pi$. $152.4 \mathrm{kHz}$. Although the calculation is done with 10 basis states the dynamics in Fig. 3] shows full two-level Rabioscillations as one would expect. A fit of the molecular state population to a $\sin ^{2}(\Omega / 2 \cdot t)$ function shows that the Rabi frequency is $\Omega=2 \pi \cdot 1.84 \mathrm{kHz}$ which exactly matches the value of the Rabi frequency displayed in Fig. 2 Matching the oscillation time such that $\Omega t=\pi$ one can transform the atomic pair into a molecule with unit probability, and using the interaction time as a control 


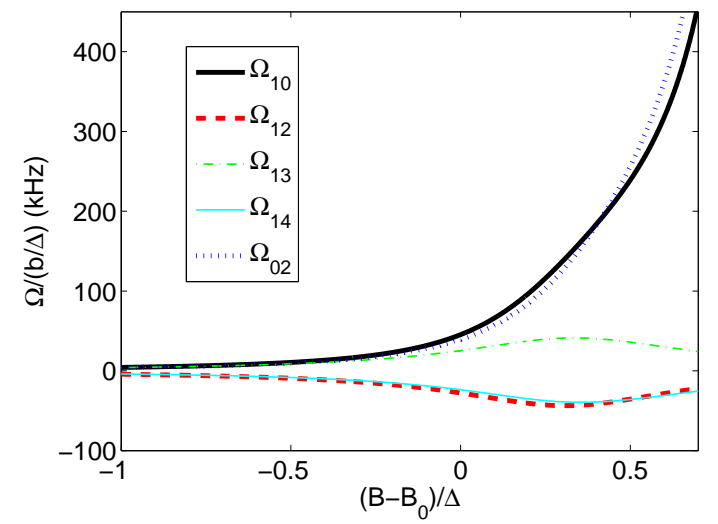

Figure 2: Amplitude normalized Rabi frequencies on resonance for ${ }^{85} \mathrm{Rb}$. The parameters are as in Fig. 11 The states are labelled $0,1,2, \ldots$ with increasing energy. E.g., $\Omega_{10}$ is the coupling between the two lowest states.

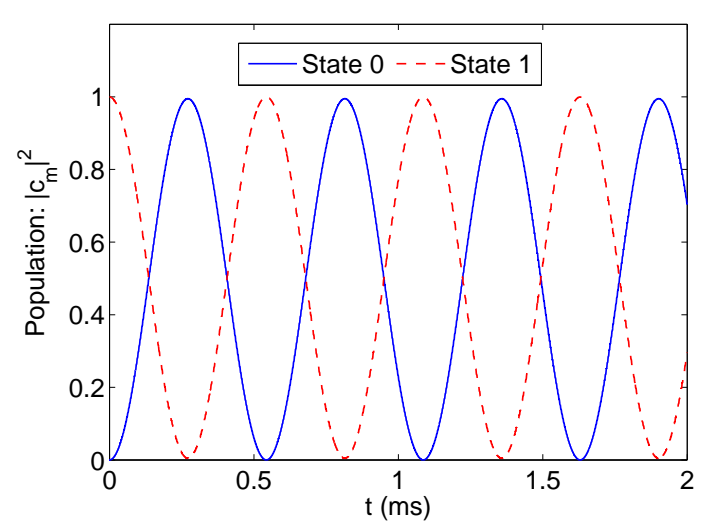

Figure 3: Dynamics of the resonant process at $B^{\prime}=B_{0}+$ $0.4 \Delta$. The field is modulated at an angular frequency $\omega_{B}=$ $2 \pi \cdot 152.4 \mathrm{kHz}$, with the amplitude $b=0.01 \Delta$. The other parameters are as in Fig. [1]

parameter, a wide range of well-controlled superposition states of the molecular and atomic component can also be prepared.

At certain magnetic fields three levels are coupled in a ladder configuration, because $\hbar \omega_{B}^{\text {res }}$ matches not only the energy distance to the molecular state, but also the distance to a higher lying atomic state (see Fig. 11). This leads to a more complicated three-level dynamics, but since the coupling to the higher lying atomic state is much smaller than the coupling to the molecular state (see Fig. 2), the higher lying atomic level becomes only sparsely populated. This is fortunate from the point of view of molecular formation. The lowest B-field on the molecular side of the resonance where such a three-level resonance occurs is $B^{\prime}=B_{0}+0.3582 \Delta=158.88 \mathrm{G}$ shown with the dashed arrows in Fig. $1\left(\omega_{B}^{\mathrm{res}}=2 \pi \cdot 125.1 \mathrm{kHz}\right)$. Simulation of the dynamics in this case shows oscillations with a peak population of $6 \%$ in the excited atomic state. All remaining population is distributed between the molecular and the lowest atomic state. At the next three-level resonance at $B^{\prime}=B_{0}+0.4385 \Delta=159.74 \mathrm{G}$ the peak population of the higher lying atomic state becomes only $4 \%$.

The scheme should work just as well for Feshbach resonances in other species like ${ }^{87} \mathrm{Rb}$ although the Feshbach resonances are more narrow and situated at higher magnetic fields. According to 25] the broadest resonance in ${ }^{87} \mathrm{Rb}$ occurs between atoms in the $\mathrm{F}=1 \mathrm{~m}_{\mathrm{F}}=1$ hyperfine state and has the following parameters: $B_{0}=1007.60(3)$ $\mathrm{G}, \Delta=0.20(3) \mathrm{G}, a_{\mathrm{bg}}=100.5 a_{0}$. This resonance has been used to produce molecules in an optical dipole trap [6] and it was also used in the recent optical lattice experiment 14]. Some Rabi frequencies for this system are shown in Fig. 目 The couplings are of the same order of magnitude, but the plot is qualitatively different from the plot in Fig. 2] The difference can be partly attributed to the different behaviour of the scattering length as $B$ approaches $B_{0}-\Delta$ (positive scattering length side in ${ }^{87} \mathrm{Rb}$ ) compared to when $B$ approaches $B_{0}+\Delta$ (positive scattering length side in ${ }^{85} \mathrm{Rb}$ ) - see (11). In the latter case the scattering length approaches 0 and the resonant frequency $\omega_{B}^{\text {res }}$ entering (9) diverges for coupling to the molecular state, because the binding energy of the molecular state in the pseudopotential model varies as

$$
E_{0}=\frac{-\hbar^{2}}{2(m / 2) a_{\mathrm{sc}}^{2}}=\frac{-\hbar^{2}}{m a_{\mathrm{bg}}^{2}\left(1-\Delta /\left(B-B_{0}\right)\right)^{2}}
$$

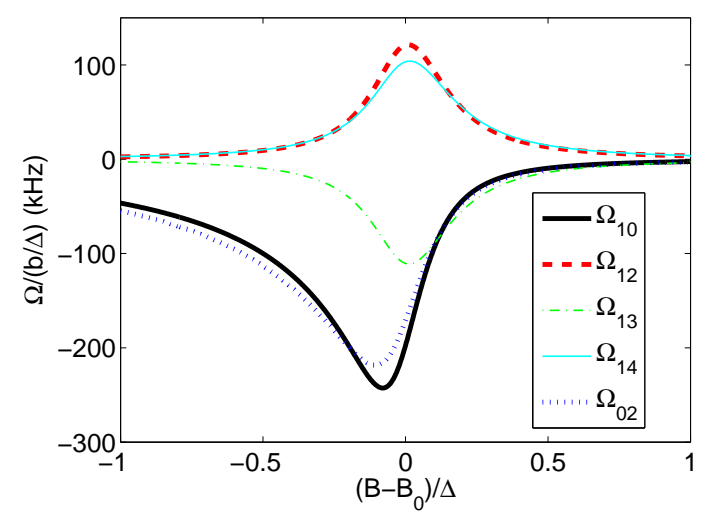

Figure 4: Amplitude normalized Rabi frequencies in ${ }^{87} \mathrm{Rb}$ (cf. Fig. 2). Here the positive scattering length (molecular) side is to the left of the resonance, because $\mathrm{a}_{\mathrm{bg}}=100.5 a_{0}>0$. We assume the same trapping frequency $\omega=2 \pi \cdot 30 \mathrm{kHz}$ as above.

Application of a $\pi$-pulse in the above scheme relies on the resonance and Rabi frequencies being exactly known and identical for all lattice sites. A more robust scheme is to sweep the frequency slowly across the resonance frequency. Using a constant amplitude linear frequency sweep from $10 \%$ below to $10 \%$ above $\omega_{B}^{\text {res }}$, we get the dynamics shown in Fig. 5 . After just a few Rabi oscillation periods the molecular state population settles near 
$100 \%$. In addition to correcting for inhomogeneity effects, a frequency sweep will also introduce some robustness against technical noise in the magnetic field value, as long as this noise is present only on longer timescales than that given by the sweeping time. This is especially interesting regarding applications to more narrow Feshbach resonances like in ${ }^{87} \mathrm{Rb}$.

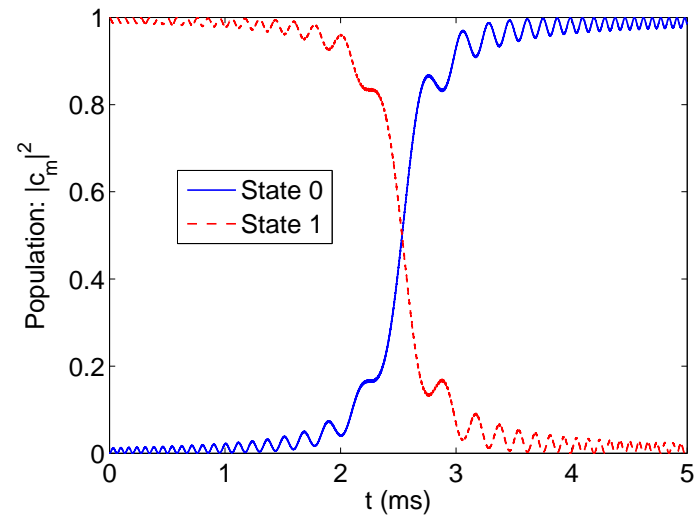

Figure 5: $5 \mathrm{~ms}$ linear frequency sweep from $10 \%$ below to $10 \%$ above the resonance frequency $\omega_{B}^{\text {res }}=2 \pi \cdot 152.4 \mathrm{kHz}$ at an applied field $B^{\prime}=B_{0}+0.4 \Delta$. The modulation has the constant amplitude: $b=0.01 \Delta$.

One might also consider the possibility of using larger oscillation amplitudes. This makes the association quicker, can be used to keep the magnitude of the harmonic modulation well above the technical noise level and gives also some robustness against a noisy value of $B^{\prime}$. Our simulations show that if the coupling strength is not much smaller than $\Delta$, the resonance frequency is significantly shifted and the oscillations are distorted, but they show a surprisingly high molecular peak population. For instance with $B^{\prime}=B_{0}+0.4 \Delta$ and $b=0.2 \Delta$ we must use a value of $\omega_{B}$ which is $45 \%$ greater than that in Fig. 3 to get maximum population oscillations, and we then get a molecular peak population of around $80 \%$ (Fig. 6). We attribute the frequency shift to the strongly increasing slope of the molecular level curve (Fig. (1) as the B-field is modulated to larger values, farther away from the resonance.

To summarize, we have analyzed the formation of molecules from atoms in an optical lattice by a resonant modulation of a B-field which causes Rabi-oscillations between the atomic and the molecular state in the vicinity of a Feshbach resonance. For identical atoms, the problem separates in a center-of-mass and a relative motion part, and the relevant s-wave scattering part was solved exactly in terms of known analytical solutions of the time-independent Schrödinger equation. Complete conversion to molecules was seen both with a $\pi$-pulse of the interaction and with a frequency swept interaction. In the case of different atoms, relevant for the formation of heteronuclear molecules, we expect the coupling between center-of-mass and relative motion to cause highly

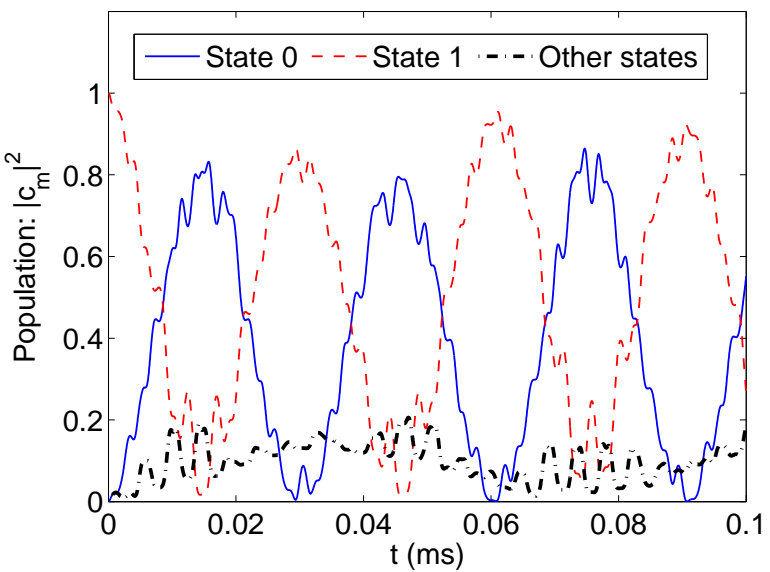

Figure 6: Dynamics of the process with a very high oscillation amplitude: $b=0.2 \Delta . B^{\prime}=B_{0}+0.4 \Delta$. The resonance frequency has shifted $45 \%$ compared to the value in Fig. 3] so $\omega_{B}=1.45 \cdot 2 \pi \cdot 152.4 \mathrm{kHz}$. The frequency of the population oscillation is $33 \mathrm{kHz}$ whereas the Rabi frequency predicted from (9) is $37 \mathrm{kHz}$. There are 20 basis states included in the calculation.

non-trivial effects.

In the experiment [8] the large number of ${ }^{85} \mathrm{Rb}$ atoms is not pairwise confined in a lattice potential, and rather than perfect Rabi oscillations between atoms and molecules, the experiments show small rapid oscillations, dying out towards a steady state with about $30 \%$ of the atoms converted into molecules. Many-body effects, including collisions in the gas, probably cause the discrepancy with the simple two-atom model. The simple picture remains, however, useful for the identification of the resonant process in the system, and we imagine that the swept modulation frequency, proposed in this article, may lead to enhanced molecular fractions in the manybody system.

Since efficient conversion into molecules inside an optical lattice was recently demonstrated by an adiabatic sweep of the B-field across the full ${ }^{87} \mathrm{Rb}$ Feshbach resonance [14], let us conclude by pointing out some relevant features of the modulated field approach. Our approach is equivalent to driven two-level transitions in laser and NMR spectroscopy, and by varying the phase, amplitude and duration of the applied B-field modulation, we have more degrees of freedom to control the transition process. For a number of quantum optics studies, e.g., for quantum non-demolition photon counting and non-classical state preparation and for quantum state tomography, the coherent Rabi oscillation phenomenon is extremely useful, and analogous proposals for trapped atoms [26] can be implemented with the modulated B-fields. In connection with applications to quantum information, unitary operations are requested that can be applied to unknown initial quantum states, and for this purpose single pulses, or possibly composite pulse sequences with builtin robustness against frequency and amplitude errors, are 
more versatile than the adiabatic passage across the full resonance.

\section{Acknowledgments}

The Authors would like to thank Michael Budde, Nico- lai Nygaard, and Uffe Vestergaard Poulsen for comments on the manuscript.
[1] E. Hodby, S. T. Thompson, C. A. Regal, M. Greiner, A. C. Wilson, D. S. Jin, E. A. Cornell, and C. E. Wieman, Phys. Rev. Lett. 94, 120402 (2005).

[2] J. Herbig, T. Kraemer, M. Mark, T. Weber, C. Chin, H.-C. Nägerl, and R. Grimm, Science 3011510 (2003).

[3] K. Xu, T. Mukaiyama, J. R. Abo-Shaeer, J. K. Chin, D. E. Miller, and W. Ketterle, Phys. Rev. Lett. 91, 210402 (2003).

[4] M. Greiner, C. A. Regal, and D. S. Jin, Nature 426537 (2003).

[5] S. Jochim, M. Bartenstein, A. Altmeyer, G. Hendl, S. Riedl, C. Chin, J. H. Denschlag, and R. Grimm, Science 3022101 (2003).

[6] S. Dürr, T. Volz, A. Marte, and G. Rempe, Phys. Rev. Lett. 92020406 (2004).

[7] A. J. Moerdijk, B. J. Verhaar, and A. Axelsson, Phys. Rev. A 51, 4852 (1995).

[8] S. T. Thompson, E. Hodby, and C. E. Wieman, Phys. Rev. Lett. 95, 190404 (2005).

[9] T. Rom, T. Best, O. Mandel, A. Widera, M. Greiner, T. W. Hänsch, and I. Bloch, Phys. Rev. Lett. 93073002 (2004).

[10] C. Ryu, X. Du, E. Yesilada, A. M. Dudarev, S. Wan, Q. Niu, and D.J. Heinzen, cond-mat/0508201 (2005).

[11] B. Damski, L. Santos, E. Tiemann, M. Lewenstein, S. Kotochigova, P. Julienne, and P. Zoller, Phys. Rev. Lett. 90110401 (2003).

[12] H. Moritz, T. Stöferle, K. Günter, M. Köhl, and T. Esslinger, Phys. Rev. Lett. 94, 210401 (2005).

[13] G. Orso, L. P. Pitaevskii, S. Stringari, and M. Wouters,
Phys. Rev. Lett. 95, 060402 (2005).

[14] G. Thalhammer, K. Winkler, F. Lang, S. Schmid, R. Grimm, and J. H. Denschlag, cond-mat/0510755 (2005).

[15] N. R. Claussen, S. J. J. M. F. Kokkelmans, S. T. Thompson, E. A. Donley, E. Hodby, and C. E. Wieman, Phys. Rev. A 67 R060701 (2003).

[16] S. L. Cornish, N. R. Claussen, J. L. Roberts, E. A. Cornell, and C. E. Wieman, Phys. Rev. Lett. 851795 (2000).

[17] M. Greiner, O. Mandel, T. Esslinger, T. W. Hänsch, and I. Bloch, Nature 41539 (2002).

[18] T. Esslinger and K. Mølmer, Phys. Rev. Lett. 90160406 (2003).

[19] K. Huang, Statistical Mechanics (John Wiley \& Sons, 1963).

[20] E. L. Bolda, E. Tiesinga, and P. S. Julienne, Phys. Rev. A 66, 013403 (2002).

[21] E. Tiesinga, C. J. Williams, F. H. Mies, and P. S. Julienne, Phys. Rev. A 61063416 (2000).

[22] B. Borca, D. Blume, and C. H. Greene, New J. Phys. 5 111 (2003).

[23] T. Busch, B.-G. Englert, K. Rzazewski, and M. Wilkens, Found. Phys., 28549 (1998).

[24] A. P. Prudnikov, Yu. A. Brychkov, and O. I. Marichev, Integrals and Series (Gordon and Breach Science Publishers, 1990), Vol. 3 formulas 2.19.23.3 and 2.19.23.4.

[25] T. Volz, S. Dür, S. Ernst, A. Marte, and G. Rempe, Phys. Rev. A 68 R010702 (2003).

[26] K. Mølmer, Phys. Rev. Lett. 90110403 (2003). 